
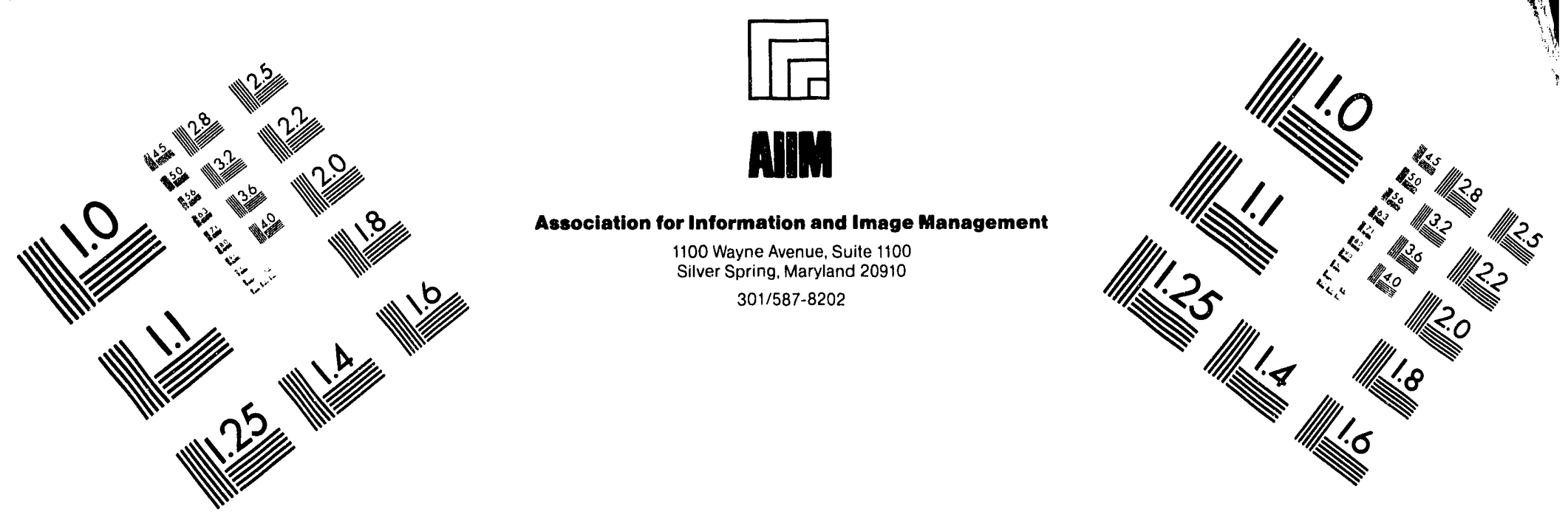

\title{
Centimeter
}

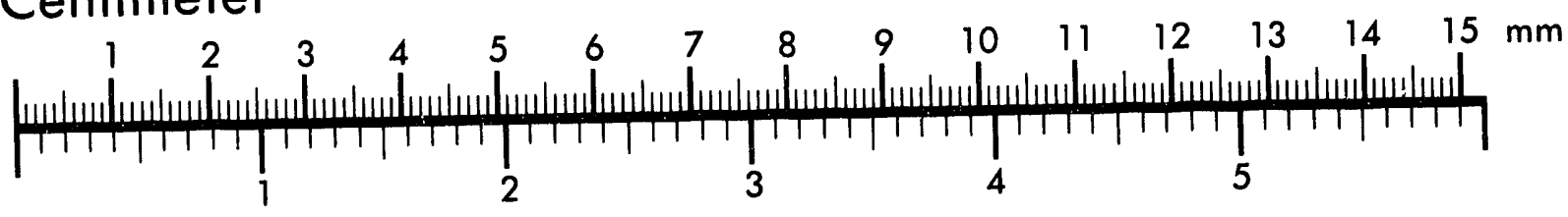
Inches
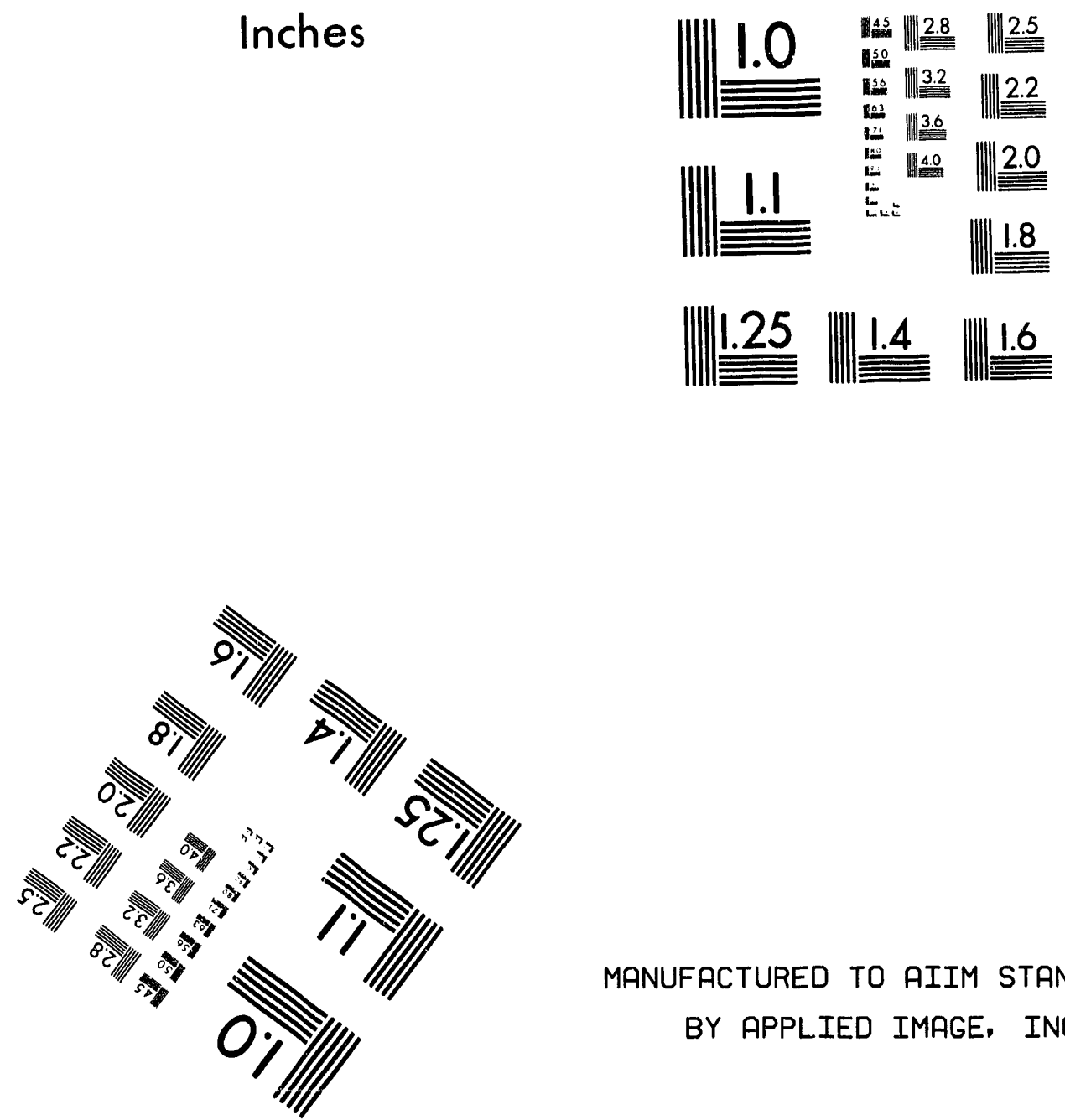

MANUFACTURED TO AIIM STANDARDS

BY APPLIED IMAGE, INC.

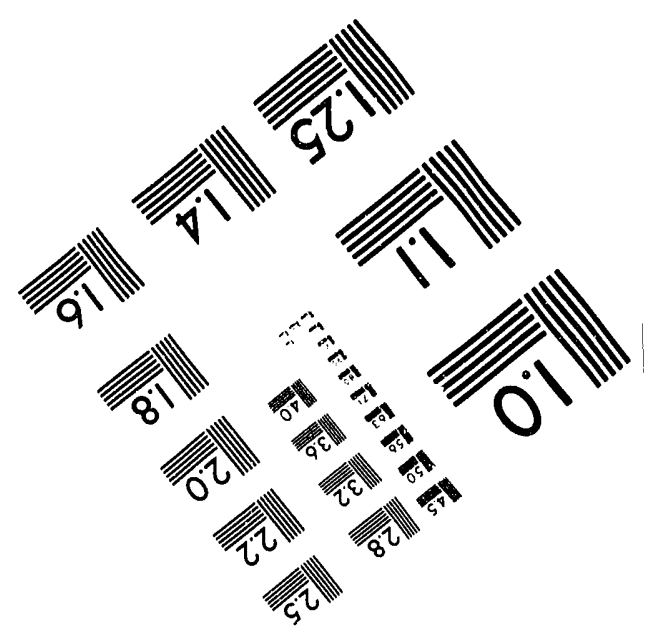



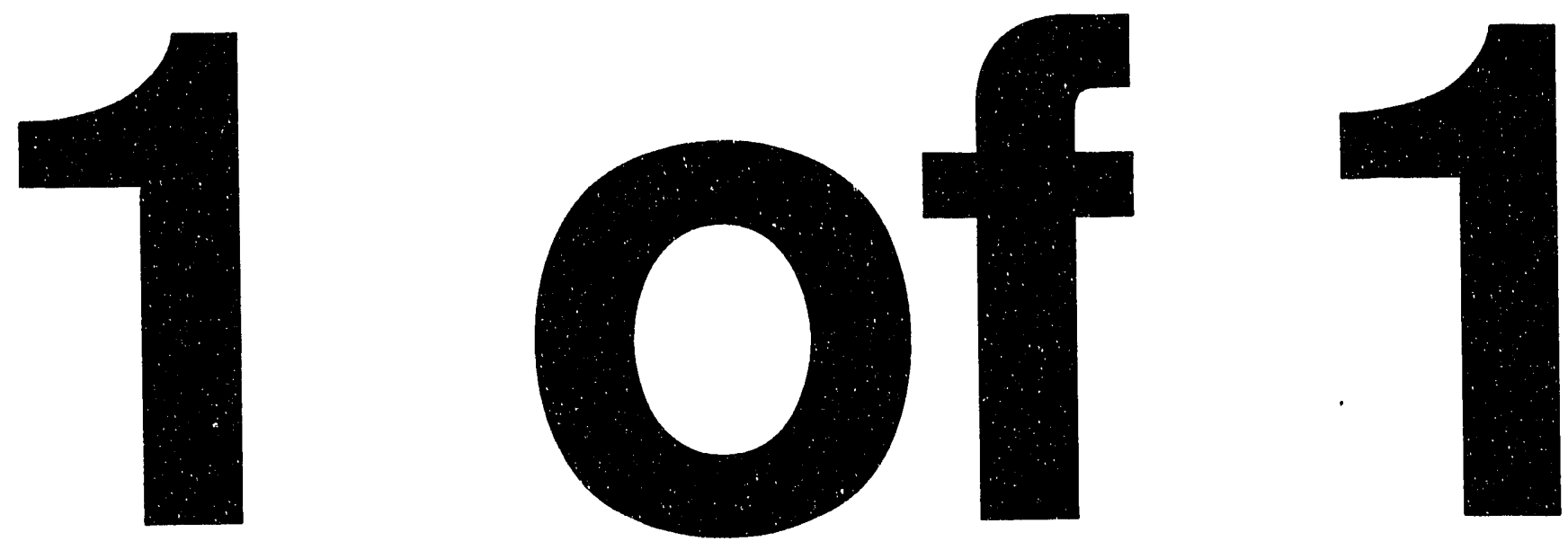


\section{TITLE: \\ A MICROSECOND-PULSEWIDTH, INTENSE, LIGHT-ION BEAM ACCELERATOR}

AUTHORS:

SUBMITTED TO:

9th IEEE Pulsed Power Conference

Albuquerque, NM

June 21-23, 1993

D. J. Rej, R. R. Bartsch, H. A. Davis,

J. B. Greenly, and W. J. Waganaar

NATIONA

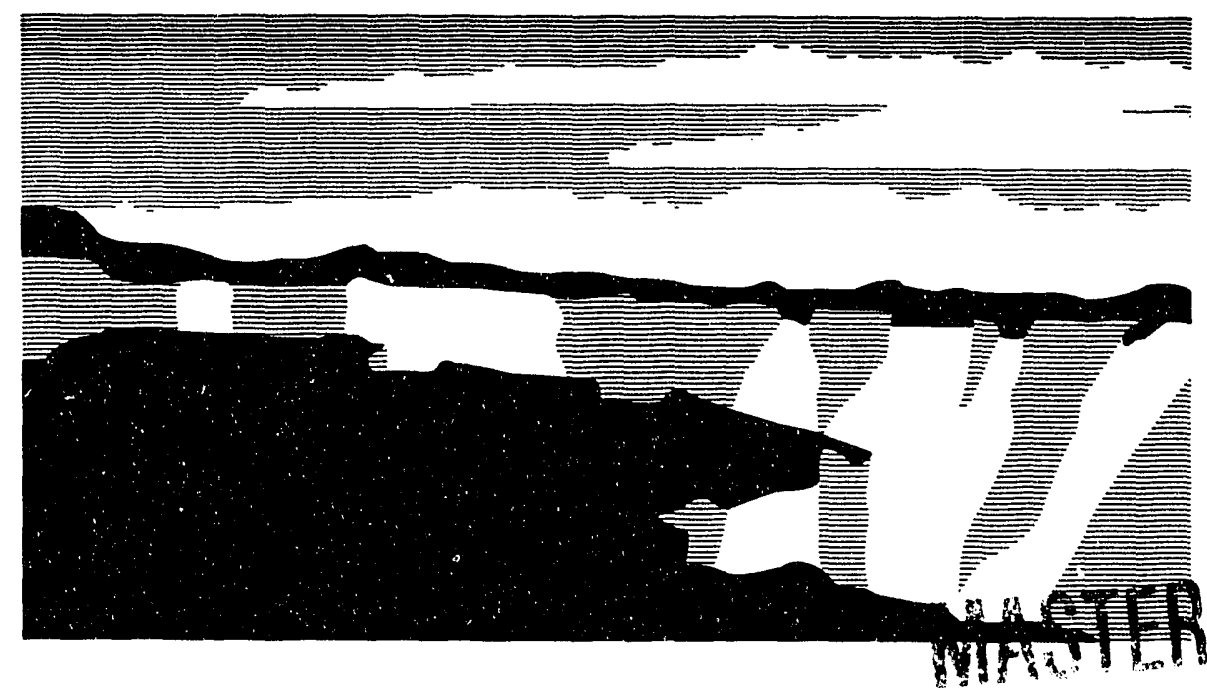

Los Alamos National Laboratory, an affirmative action/equal opportunity emplóyer, is operated by the University of California for the U.S. Department of Energy under contract W-7405-ENG-36. By acceptance of this article, the publisher recognizes that the U.S. Government retains a nonexclusive, royalty-free ticense to publish or reproduce the published form of this contribution, or to allow others to do so, for U.S. Government purposes. The Los Alamos National Laboratory requests that the publisher identify this article as work performed under the auspices of the U.S. Department of Energy. 


\title{
A MICROSECOND-PULSEWIDTH, INTENSE, LIGHT-ION BEAM ACCELERATOR
}

\author{
D. J. Rej, R. R. Bartsch, H. A. Davis, J. B. Greenly,* W. J. Waganaar \\ Physics Division, MS-E526, Los Alamos National Laboratory, Los Alamos, NM 87545
}

\begin{abstract}
A relatively long-pulsewidth $(0.1-1 \mu \mathrm{s})$ intense ion beam accelerator has been built for materials processing applications. An applied- $\mathrm{B}_{\mathrm{r}}$, magnetically-insulated extraction ion diode with dielectric flashover ion source is installed directly onto the output of a $1.2-\mathrm{MV}, 300-\mathrm{kJ}$ Marx generator. Initial operation of the accelerator at $0.4 \mathrm{MV}$ indicates satisfactory performance without the need for additional pulse-shaping.
\end{abstract}

\section{Introduction}

Over the last two decades, there has been remarkable progress in the understanding and development of intense, pulsed ion beams. ${ }^{1,2}$ The primary application driving the development of this technology is inertial confinement fusion (ICF) energy research. ${ }^{3}$ Recently, however, several novel materials processing applications utilizing intense ion beam technology have emerged. Because of the short range of ions in matter, applications usually involve the surface modification of materials, e.g., implantation, ${ }^{4}$ alloy mixing, 5,6 defect formation, ${ }^{7,8}$ polishing, ${ }^{9}$ glazing, ${ }^{9,10}$ and thin film deposition. ${ }^{11-14}$ For many of these applications, the beam acts as a heat source that will rapidly melt or evaporate a target material surface. Consequently, long-pulsewidth intense ion beams are desirable to maximize surface heating with minimum shock waves, provided that thermal conduction into the target during the beam pulse is low. For most materials and $1-\mathrm{MeV}$ beam energies, microsecond-long pulses may be optimal. Thus, materials processing applications could be enhanced by the development of beam pulsewidths greater than the 10-50 ns needed for ICF. In this paper, we report details of a long-pulsewidth, intense, light ion beam driver constructed at Los Alamos National Laboratory, which utilizes an applied- $B_{r}$, magnetically-insulated ion diode that is installed directly onto the output of a Marx generator.

The ion diode geometry is shown schematically in Figure 1. Ions are accelerated by a high-voltage pulse applied across an annular anode-cathode (A-K) gap located in vacuum. Undesirable currents from field-emitted cathode electrons are suppressed by an applied radial magnetic field $B_{r}$. The field is generated by pulsed electromagnets (shown in the schematic as two concentric coils on the cathode side) and flux-excluding metal components located in the vacuum chamber. The electrons are confined in a sheath, thereby forming a virtual cathode which can enhance the local accelerating electric field and reduce the ion space charge; consequently, ion diodes are intrinsically high-power-density devices, capable of operating with ion current density enhancement factors 10 to 100-times the Child-Langmuir space-charge limit for the vacuum condition. 15,16 The applied- $\mathrm{B}_{\mathrm{r}}$ configuration in Fig. 1 is also known as an "extraction diode" since the accelerated beam can exit the diode region; subsequently, the beam may be focussed and propagated, provided there are sufficient space-chargeneutralizing electrons downstream. The cylindrically-symmetric geometry is desirable since the electron $\mathbf{E} \times \mathbf{B}$ drift trajectories may remain confined in the A-K gap, thereby minimizing the electron current and enhancing the ion current density. For optimum performance with the dielectric anode ion sources used here, $B_{r}$ is usually set to about 1.5-times the critical field needed to confine the accelerated electrons in the A-K gap. The extraction geometry is attractive since relatively long ion beam pulsewidths, desirable for several materials processing applications, may be obtained. In contrast, non-axisymmetric diode configurations have large asymmetric plasma production caused by electron bombardment along one side of the A-K gap. ${ }^{17}$ Expansion of this plasma may prematurely close the gap, thus limiting the overall useable pulsewidth.

A relatively simple and common anode ion source uses the surface breakdown of a solid dielectric attached to a metallic anode. Sheath electrons diffuse across the A-K gap from the cathode and bombard the anode surface to produce a plasma. Plasma generation is believed to be initiated by dielectric breakdown induced by the excess negative charge of accelerated electrons which accumulate in the anode surface. More advanced ion sources have also been developed. For example, anodes constructed from condensed gases on cryogenic surfaces, ${ }^{18}$ and gas-loaded metallic foils, ${ }^{19}$ have resulted in high-purity proton and deuteron beams. Also, active plasma sources using localized gas injection and breakdown before application of the accelerating voltage have been developed to produce a pure, reproducible beam capable of high repetition rate and long hardware lifetime. ${ }^{20}$

The intense ion beam device discussed in this paper is an extension of earlier work involving microsecond pulsewidth accelerators, which were developed for magnetic fusion applications and utilized both the extraction diode geometry at 100$\mathrm{kV}$ voltages, ${ }^{21}$ and the cylindrical "barrel diode" configuration at 1 MV voltages. 22

\section{Ion Diode Design}

The extraction diode that has been developed is illustrated in Figure 2. The magnet coils are positioned on the cathode side, rather than on the anode side, for several reasons. First, the cathode is electrically grounded, so there is no need for highvoltage isolation of the coils from their power supply. Second, electrons field emitted from the cathode tips connect to field lines which do not intersect high-voltage anode structures (Fig. 3). This is important to avoid electron leakage currents and to build up an adequate virtual cathode in the A-K gap. Third, there is minimum net magnetic deflection of ions. The beam propagates through the return magnet flux, thereby conserving canonical angular momentum. In other words, the impulse imparted by the return flux counteracts the $\mathbf{v}_{\mathbf{z}} \times \mathbf{B}_{\mathbf{r}}$ force imparted on the ions as they accelerate across the A-K gap. A potential problem with this configuration is in the magnetized drift region between cathode cylinders. Electrons remain magnetically insulated and are not free to stream with the ion beam in this region; thus, space-charge neutralization must be provided, for example by external gas injection 23 or from wall emission.

The dielectric "flashover" anode consists of a Lucite annulus, 194-mm i.d., 292-mm o.d., 6.4-mm thick, mounted concentrically onto an aluminum high-voltage electrode. Both solid and perforated (with 712 evenly-spaced, $0.8-\mathrm{mm}$ diameter holes) anodes have been used. Based on previous operating experience, the beam constituency is expected to be a mixture of the elements found in the anode, $\mathrm{H}, \mathrm{C}$, and $\mathrm{O}$. The cathode consists of two concentric cylinders constructed from Ti-8Al-1Mo-1V alloy, each $102-\mathrm{mm}$ 


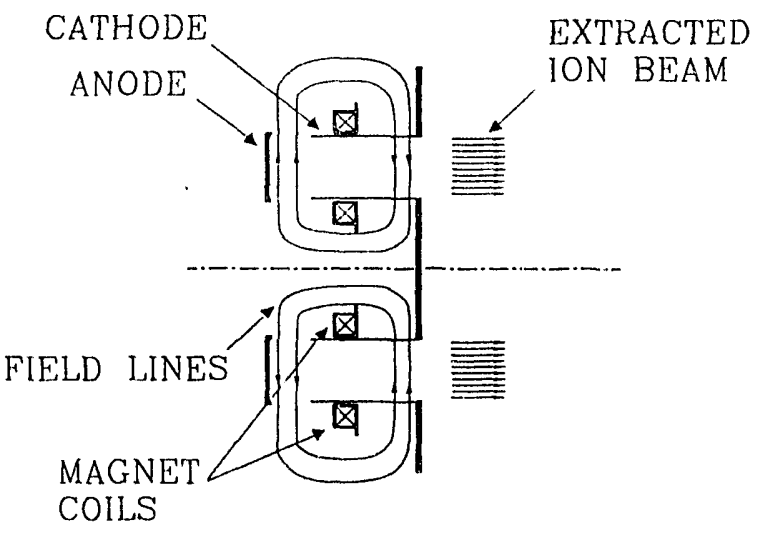

Fig. 1: Schematic drawing of an applied-B $B_{r}$ extraction diode.

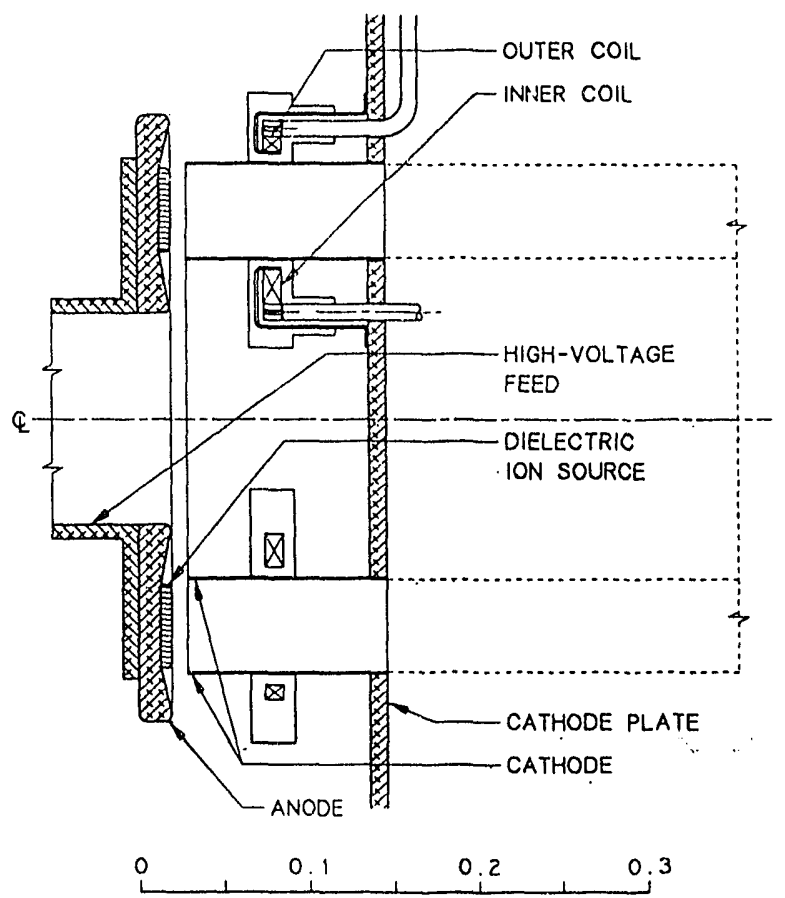

Fig. 2: Intense ion beam diode design. Scale is in meters.

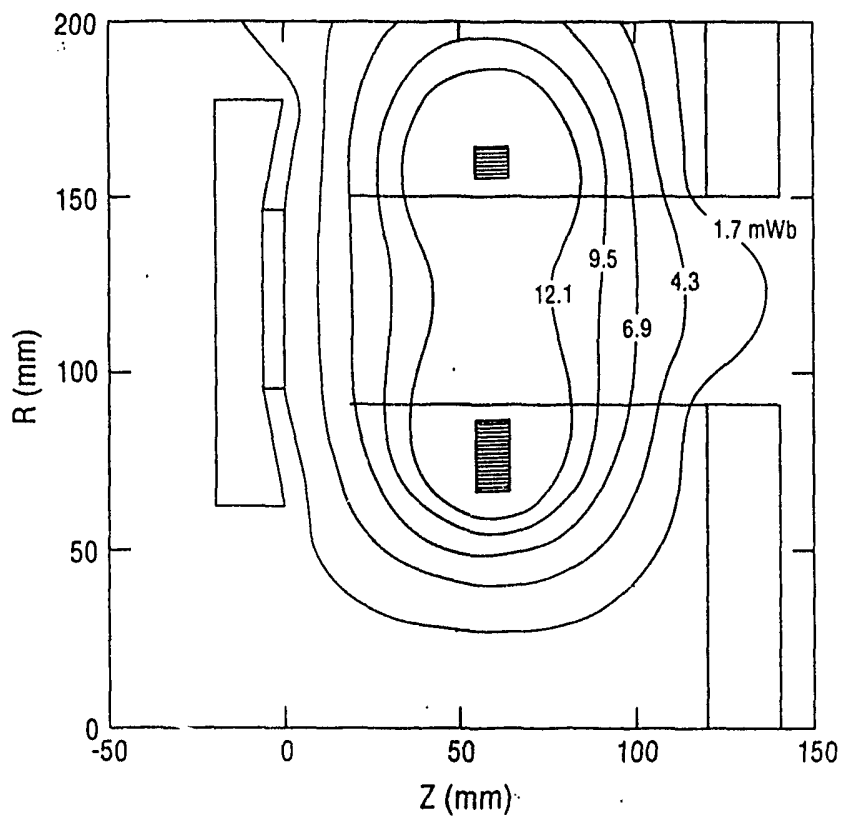

Fig. 3: Vacuum magnetic field flux surfaces in ion diode. long and $0.8-\mathrm{mm}$ thick with diameters of $186 \mathrm{~mm}$ and $298 \mathrm{~mm}$. For these experiments, the anode-cathode gap spacing is $19 \mathrm{~mm}$.

The $B_{r}$ field is produced by two concentric magnets, 18 turns with a $77-\mathrm{mm}$ mean radius (inner) and 7 turns with $161-\mathrm{mm}$ radius (outer), positioned midway between the anode and an aluminum plate on which the magnets and cathode electrodes are mounted. Each magnet is wound from $0.5-\mathrm{mm}$ thick, $10-\mathrm{mm}$ wide berylliumcopper tape 24 which is wrapped with $0.2-\mathrm{mm}$ thickness Kapton film $(0.05-\mathrm{mm}$ thick, $13-\mathrm{mm}$ wide film, wrapped in two layers, half lapped) for electrical insulation. The $\mathrm{BeCu}$ tape is obtained fully annealed to enable easy wrapping and winding. After being wound, the coils are precipitation hardened in a vacuum oven at $400^{\circ} \mathrm{C}$ for four hours. Following heat treatment, the high-voltage leads (consisting of the center conductor and insulation of RG $217 / \mathrm{U}$ cable) are soldered to the coil. The coils are then wrapped with fiberglass cloth, while the leads are enforced with NEMA G10 composite. Each magnet assembly is then individually cast in epoxy. 25

The magnets are independently powered by $10-\mathrm{kV}, 500-\mu \mathrm{F}$ capacitors. The coil current risetimes are $170 \mu$ s (outer) and $250 \mu \mathrm{s}$ (inner), which are sufficiently long for fields to penetrate the cathode electrodes, but short enough to prevent significant penetration into the aluminum anode electrode. Typical computed vacuum magnetic field flux surfaces and field profiles are shown in Figures 3 and 4, respectively, for $6 \mathrm{kA}$ currents in both coils.

The diode is configured within a vacuum enclosure consisting of aluminum (6061/T6) and stainless-steel (304) chambers. A $2 \times$ $10^{-6}$ torr base pressure is obtained with a $0.4-\mathrm{m}$ cryopump. As illustrated in Figure 5, the evacuated assembly may be subdivided into five regions: (1) the high-voltage interface to the Marx generator; (2) the vacuum pump stand; (3) a 0.3-m-long spool that houses an optional plasma opening switch (POS); (4) the applied- $\mathrm{B}_{\mathrm{r}}$ ion diode enclosure; and (5) the materials-processing chamber. A photograph of the assembled accelerator is shown in Figure 6.

\section{High-Voltage Power Supply}

The ion diode is powered by a 10-stage Marx generator with a $300-\mathrm{kJ}$ output at 1.2 MV. The Marx generator consists of sixty $2.8-\mu \mathrm{F}, 60-\mathrm{kV}$ capacitors, configured 3 units in parallel to a pack, and 20 packs in series. The entire assembly is contained in dielectric oil for high-voltage insulation. Bipolar charging results in a $120-\mathrm{kV}$ spark gap voltage. All ten spark gaps are triggered by a single $280-\mathrm{kV}, 0.8-\mathrm{kJ}$ trigger Marx generator.

The diode is connected directly to the Marx generator through a conventional aluminum/Lucite stacked-ring oil-vacuum interface as shown in Fig. 5. There are no output switches or pulse-forming

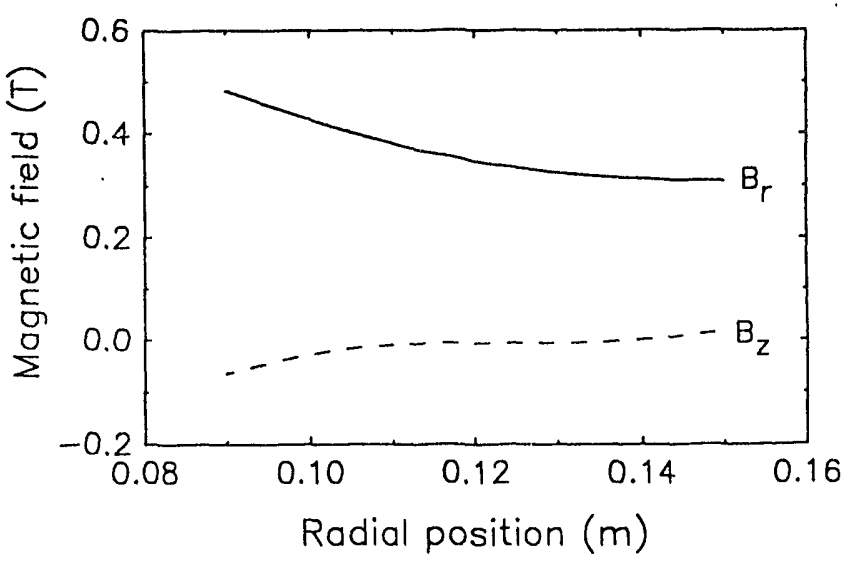

Fig. 4: Magnetic field as a function of radial position inside A-K gap. 


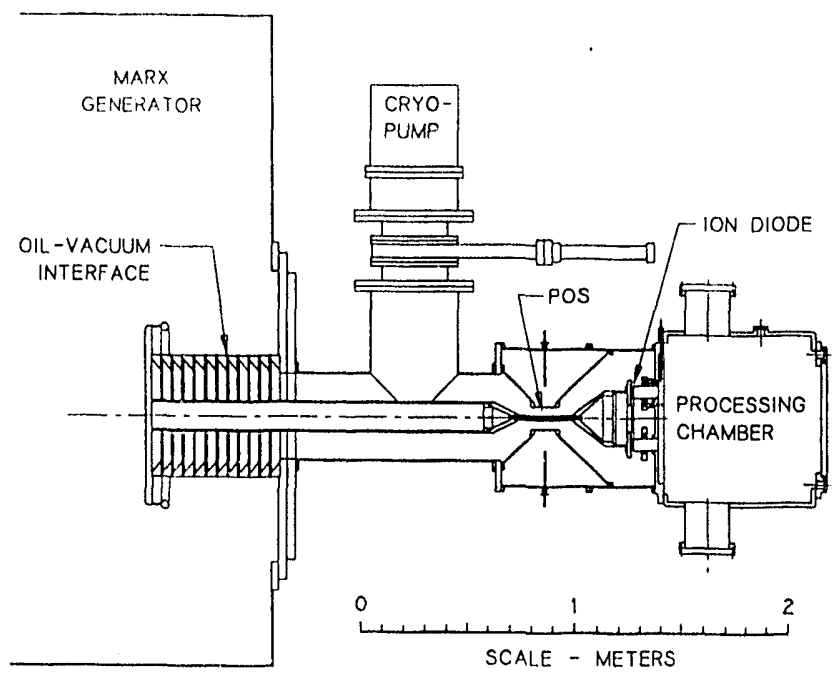

Fig. 5: Assembly drawing of ion beam generator.

lines, although a plasma opening switch (POS) has been constructed for future experiments to shape the diode voltage pulse. The combined source inductance of the Marx/interface/diode configuration is approximately $3 \mu \mathrm{H}$. A $1.6-\Omega$ series resistance is installed between the Marx output and the vacuum interface to limit the output current after A-K gap closure.

\section{Initial Performance}

Initial experiments have been performed at Marx erection voltages of approximately $600 \mathrm{kV}$. Diagnostics include a resistive voltage divider and Rogowski coil, both located near the oilvacuum interface, to measure Marx voltage and total output current, respectively. These monitors were built and calibrated by the manufacturer ${ }^{26}$ of the oil-vacuum interface. The $10^{4}: 1$ voltage divider was calibrated to $\pm 3 \%$ accuracy. The Rogowski coil was calibrated in-situ with its passive integrator to $\pm 7 \%$.
Ion current densities $j_{i}$ are measured with a radial array of four Faraday cups placed near one azimuthal location at an axial position $138 \mathrm{~mm}$ from the anode. The Faraday cup design utilizes a recommended geometry, 27 and consists of a Elkonite collector aligned behind a $0.5-\mathrm{mm}$-diameter entrance aperture. The collector detects ions over a $394 \mathrm{mrad}$ acceptance angle which is about twotimes larger than typical beam divergences. To suppress electrons that stream with the ion beam. the collector has been operated at negative bias voltages up to $600 \mathrm{~V}$. The theories of biased Faraday cup operation for intense ion beams reveal that secondary electron emission from the collector to the grounded housing may be neglected.27-29 The biased collectors, however, are found unreliable for our accelerator. At approximately 100 to 200 ns into the pulse, a breakdown between collector and ground is observed, presumably caused by a plasma ablated off the collector by the beam. This breakdown results in an erroneously high Faraday cup signal. Electron currents have been suppressed without breakdown on unbiased collectors when a 0.2-T transverse magnetic field is applied over a region $\pm 1 \mathrm{~cm}$ around the entrance aperture. The field is produced by two SmCo permanent magnets positioned on the outer surface of the Faraday cup housing. Several phenomenon can cause errors in Faraday cup measurements. For example, $j_{i}$ may be underestimated if electrons are not completely filtered from the beam, or if a high-density plasma ablated near the entrance aperture attenuates ions. Conversely, a more tenuous plasma can strip non-hydrogenic ions resulting in an overestimate in $j_{i}$. Unfortunately, a realistic absolute calibration of an intense ion beam Faraday cup is difficult, given the high power density, 100 $\mathrm{GW} / \mathrm{m}^{2}$ or more, of the ion bearn. For confidence in this diagnostic, one must rely on earlier conparisons of similar Faraday cup designs with other calibrated beam diagnostics such as particle spectroscopy and Rogowski belt measurements. ${ }^{29}$ These comparisons typically yield overall confidence levels ranging between \pm 20 and $\pm 40 \%$.

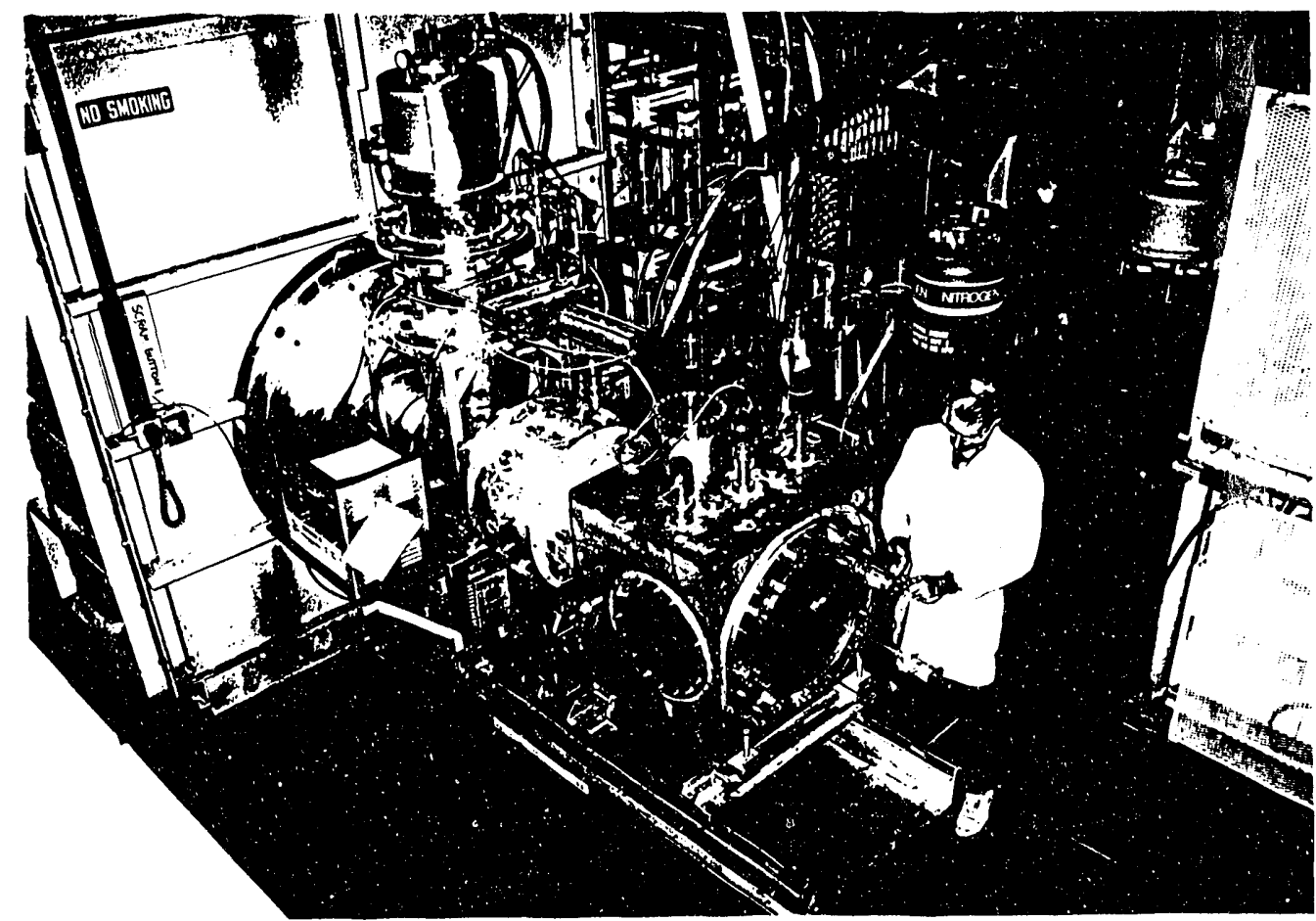

Fig. 6: Photograph of the intense ion beam device. 


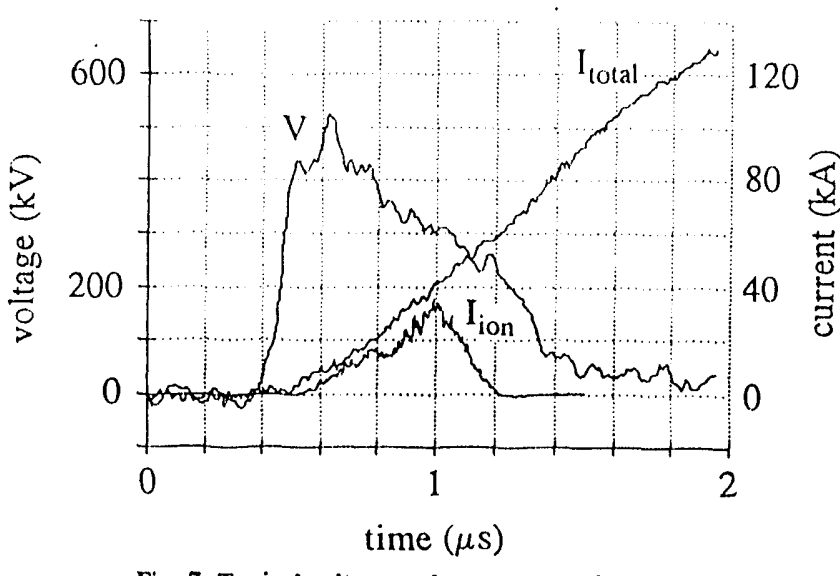

Fig. 7: Typical voltage and current waveforms.

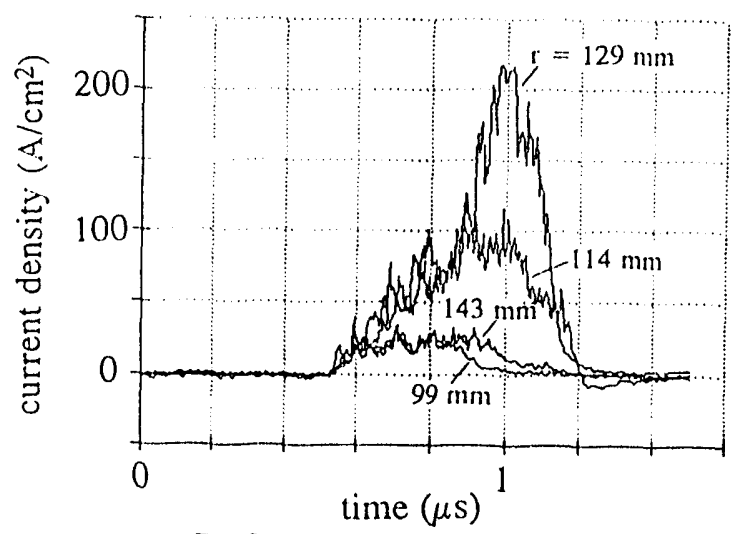

Fig. 8: Typical Faraday cup signals.

Typical waveforms for the diode voltage (with inductive correction) $V$ and current $I$ from a single pulse are shown in Figure 7. The cumulative errors arising from diagnostic and digitizer calibration and noise are estimated at $\pm 7 \%$ for $V$ and $\pm 9 \%$ for $I$. Initially, the diode impedance is high, resulting in a voltage risetime that is less than 100 ns. Faraday cup traces are plotted in Figure 8 for the same pulse. Ions are detected starting 200 ns after application of the high-voltage. Ion emission is terminated approximately $600 \mathrm{~ns}$ later, presumably due to short circuiting of the diode by expansion of an anode plasma or cathode sheath. The ion emission pulsewidth implies a plasma closure speed of about 25 $\mathrm{mm} / \mu \mathrm{s}$, which is consistent with that observed in other devices. ${ }^{30,31}$ The peak ion current of $33 \mathrm{kA}$ is inferred from the product of the anode area and the radially-weighted average of the Faraday cup signals. Further details on the initial ion diode performance may be found elsewhere. ${ }^{13}$

* Permanent Address: Cornell University, Ithaca, NY 14853

\section{Acknowledgment}

This research is funded by the USDOE through the Laboratory Directed Research and Development Program at Los Alamos.

\section{References}

[1] V. M. Bystritskii, and A. N. Didenko, High-Power lon Beams (American Institute of Physics, New York, 1989).

[2] R. N. Sudan, in Inertial Confinement Fusion, A. Caruso and E. Sindoni Eds. [Intern. School of Plasma Physics "Piero Caldirola", Italian Phys. Soc., Bologna, 1989], p. 453.

[3] J. VanDevender, D. Cook, Science 232, 831 (1986); J. VanDevender, Plasma Phys. and Contr. Fusion 28, 841 (1986).
[4] W. Chu, et. al. . Nucl. Instr. and Meth. 194, 443 (1982).

[5] R Fastow and J. W. Mayer, J. Appl. Phys. 61, 175 (1987).

[6] Y. Nakagawaet. al., Nucl. Instr. and Meth. B39, 603 (1989).

[7] A. Progrebnyak et. al., Phys. Stat. Sol. A 123, 119 (1991).

[8] A. N. Didenkoet. al., Mat. Sci. and Eng. A115, 337 (1989).

[9] R. W. Stinnett et. al., in Proc. 1993 IEEE Intern. Conf. on Plasma Science (IEEE, Piscataway, NJ, 1993) p. 114

[10] D. S. Phillips, G. P. Johnston, D. J. Rej, R. W. Stinnett, W. J. Waganaar, to be publlished.

[11] Y. Shimotori, M. Yokoyama, H. Isobe, S. Harada, K. Masugata, and K. Yatsui, J. Appl. Phys. 63, 968 (1988).

[12] O. I. Goncharov et. al., in Proc. 8th Intern. Conf. on itighPower Particle Beams, B. N. Breizman and B. A. Knyazev Editors (World Scientific Publishing Co., Teaneck, NJ, 1991), Vol. II, p. 1243.

[13] D.J. Rej et. al.,in Proc. 9th Intern. Conf. on High-Power Particle Beams, V. Granestein and D. Mosher Editors (Univ. of Maryland, 1992) in press.

[14] D. D. Hinshelwood et. al., inProc. 1993 IEEE Intern.Conf. on Plasma Science (IEEE, Piscataway, NJ, 1993) p. 116

[15] Y. Nakagawa, Japanese J. Appl. Phys. 23, 643 (1984).

[16] D. J. Johnson, J. P. Quintenz, and M. A. Sweeny, J. Appl. Phys. 57, 794 (1985).

[17] K. Yatsui et. al., Lasers and Particle Beams 3, 119 (1985).

[18] K. Kasuya et. al.,in Proc. 8th Intern. Conf. on High-Power Particle Beams, B. Breizman and B. Knyazev Editors (World Scientific Publishing, Teaneck, NJ, 1991), Vol. I, p. 543.

[19] H. J. Bluhm et. al., Proc. IEEE 80, 995 (1992).

[20] J. B. Greenly et. al., J. Applied Physics 63, 1872 (1988).

[21] D. A. Hammer et. al., in Proc. 6th Intern. Conf. on HighPower Particle Beams, B. Yamanaka, Editor (Osaka Univ., Osaka, Japan, 1986), p. 391.

[22] S. C. Luckhardt and H. H. Fleischmann, Appl. Phys. Lett. 30, 182 (1977).

[23] D. J. Johnson, T. R. Lockner, J. Appl. Phys. 61, 20 (1987).

[24] Alloy No. 3, available from Brush-Wellman Inc., Elmhurst, IL 60126.

[25] Magnets are cast in Stycast 1264 epoxy, available from Emerson and Cuming Inc., Woburn, MA 01888. Pot lifetimes and room-temperature cure times are 3 and 48 hours, respectively. During the casting procedure, the epoxy is mixed, vacuum degassed, and poured into the coil-mold assembly at atmospheric pressure. The assembly is then placed inside an autoclave and evacuated for approximately two hours. Castings are cured for approximately 48 hours in the autoclave at room temperature with an ambient air pressure of approximately $8 \mathrm{~atm}$.

[26] Pulsed Sciences Inc., San Leandro, CA 94577.

[27] R. Kraft and B. R. Kusse, J. Appl. Phys. 61, 2123 (1987).

[28] C. Eichenbergeret. al., J. Appl. Phys. 48, 1449 (1977).

[29] C. R. Struckman, PhD Thesis, Cornell University (1992); and D. J. Johnson, personal communication (May 1993).

[30] C. Litwin and Y. Maron, Phys. Fluids B 1, 670 (1989).

[31] Y. Kawano et. al., Lasers and Particle Beams 7, 277 (1989). 

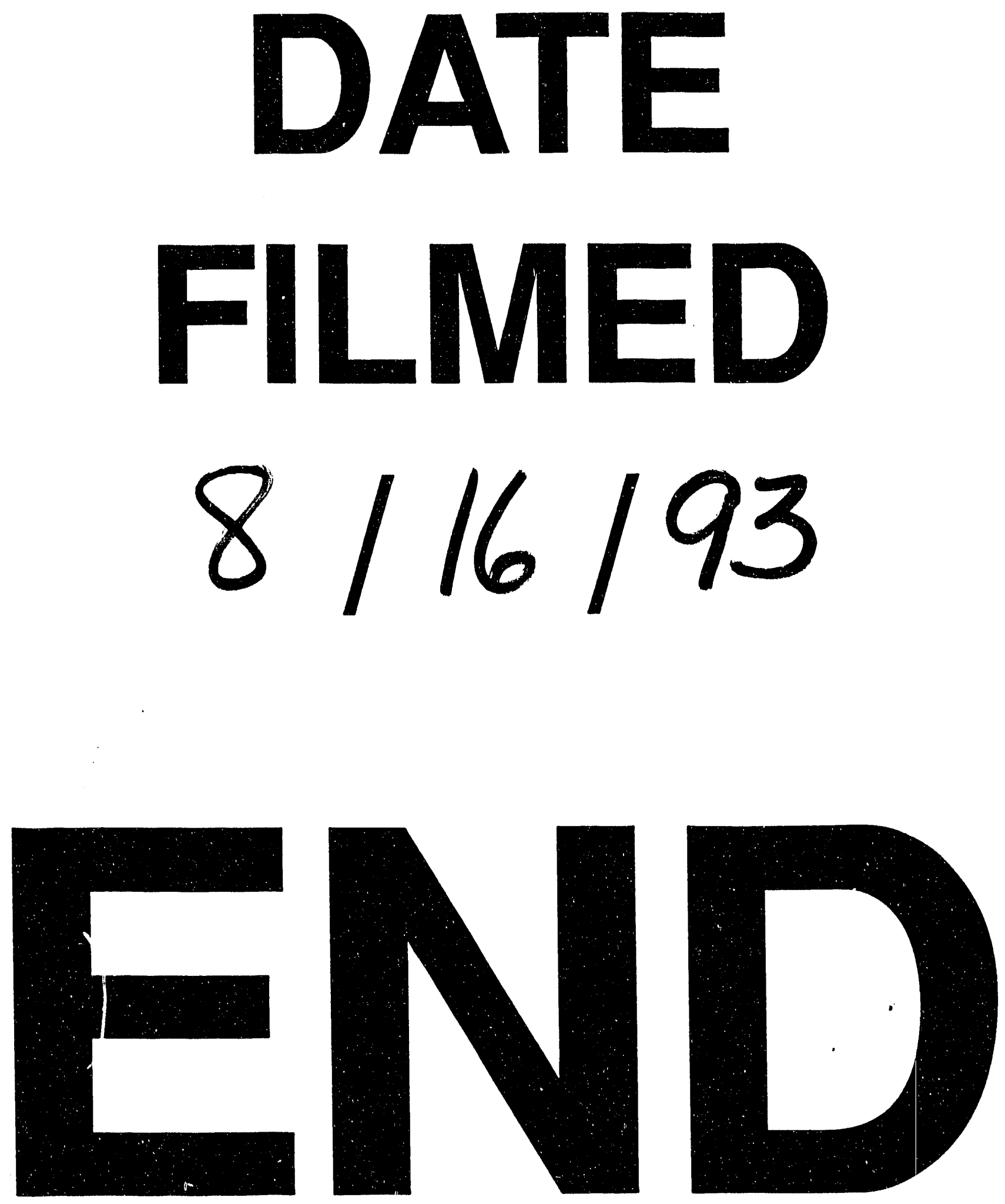
(2) 